\title{
Artelogie
}

Recherche sur les arts, le patrimoine et la littérature de l'Amérique latine

$6 \mid 2014$

Horizons et dispositifs des arts plastiques des pays du Río de la Plata (XXe siècle)

\section{Horizons et dispositifs des arts plastiques des pays du Río de la Plata (XXe siècle)}

Jacques Poloni-Simard et Edgard Vidal

\section{OpenEdition}

Journals

Édition électronique

URL : http://journals.openedition.org/artelogie/1264

DOI : 10.4000/artelogie. 1264

ISSN : 2115-6395

Éditeur

Association ESCAL

Référence électronique

Jacques Poloni-Simard et Edgard Vidal, «Horizons et dispositifs des arts plastiques des pays du Río de la Plata (XXe siècle) », Artelogie [En ligne], 6 | 2014, mis en ligne le 24 juin 2014, consulté le 24 septembre 2020. URL : http://journals.openedition.org/artelogie/1264 ; DOI : https://doi.org/10.4000/ artelogie. 1264

Ce document a été généré automatiquement le 24 septembre 2020.

Association ESCAL 


\title{
Horizons et dispositifs des arts plastiques des pays du Río de la Plata (XXe siècle)
}

\author{
Jacques Poloni-Simard et Edgard Vidal
}

\section{Horizons et résonances des arts plastiques du Río de la Plata (XXe siècle)}

\section{Jacques Poloni-Simard}

Avec ce dossier d'Artelogie, consacré aux pays du Río de la Plata au cours du xxe siècle il ne concernera par ailleurs que les arts plastiques -, on a cherché à éviter les problématiques par trop « argentino- » ou « uruguayo-centrées », celles-là même qui s'interrogent sur l'art «national » ou « international ». On ne trouvera pas non plus un panorama, même sélectif, des séquences classiques (écoles ou courants) et des chronologies canoniques (modernisme, avant-gardes, années des dictatures). Les contributions rassemblées mettent en revanche en valeur les connexions et les articulations - ce que l'on a appelé les «horizons » - avec d'autres milieux et scènes artistiques, soit la projection de l'art argentin et uruguayen "hors d'eux-mêmes". Plutôt que de se concentrer encore sur l'attraction parisienne, Diana B. Wechsler dessine une autre carte du modernisme, qui connecte entre eux l'Argentine et la France, certes, mais aussi l'Italie et l'Espagne, ainsi que le Mexique ; et Jacques PoloniSimard attire l'attention sur une autre destination, les Andes, que des artistes ont prise entre les années 1920 et les années 1950. Mais là où les œuvres sont présentées, elles sont en situation de dialogue, et leurs auteurs interagissent avec les lieux où ils travaillent. On sait que la présence artistique latino-américaine à Paris est un phénomène ancien, qui s'est prolongé bien au-delà de la Seconde Guerre mondiale. Mais plutôt que de présenter la contribution des artistes argentins à la vie artistique locale, Isabel Plante réfléchit à la façon dont ils ont intégré dans leur travail la question de leurs déracinement et implantation, et Christine Frérot nous présente une expérience d'auto-organisation, "l'Espace latino-américain de Paris ", qui fonctionna entre 1980 et 1993. Enfin, les circulations n'alimentent pas seulement un face à face entre le Río de la Plata et l'Europe; elles sont également intra-américaines et 
alimentent d'autres interactions, auxquelles l'historiographie s'intéresse depuis peu. Silvia Dolinko analyse ainsi les significations d'une exposition Antonio Berni à Montevideo en 1966, auréolé de son prix obtenu à la XXXIe Biennale de Venise en 1962 pour ses xylo-collages. D'autres articles envisagent quelques débats clés, dans le contexte argentin plus particulièrement : art et politique, art et critique sociale, art et mémoire. Or leur chambre d'écho n'est pas circonscrite à cette échelle nationale ; ces questions entrent en résonance avec bien des situations contemporaines. Référence obligée dans toute étude sur l'art conceptuel latino-américain, Ana Longoni revient sur Tucumán Arde (1968), devenu un "mythe »; mais comment récupérer sa dimension politique ? Viviana Usubiaga décrypte la réception en Argentine de la Transvanguardia pendant les années 1980 : la scène est locale, mais sa teneur critique est globale. Andrea Giunta, quant à elle, s'attache à la question de la mémoire de la dictature, à partir des défis en termes de représentation auxquels s'affrontent nombre d'artistes et des enjeux mémoriels auxquels sont confrontés les dispositifs muséographiques mis en place. Le cas argentin est ici emblématique, et sa leçon a une portée universelle.

\section{Dispositifs et dislocations : les variations platenses.}

\section{Edgard Vidal}

Un deuxième volet du dossier est porté par une interrogation sur les dispositifs et les dislocations. Dans cette perspective, l'attention est posée sur les frontières de l'activité artistique aussi bien que sur le rôle joué par des phénomènes tels que la bilocalisation d'artistes, leurs exils et leurs expatriations. Ces agencements composites et ces déplacements des hommes et des œuvres (innervés par des fractures et des errances vigoureusement créatives) s'inscrivent dans ce numéro, soit dans des problématiques ouvertes et / ou inédites, soit par la détermination de leurs démarches dans une perspective résolument plurielle de la culture latino-américaine. Trois articles sont centrés sur des expériences, individuelles ou collectives, qui montrent combien il est réducteur de rapporter œuvres et artistes aux seules catégories et classifications communément utilisées. Leurs dispositifs brouillent les frontières de l'activité plastique. Nestor M. Habkost envisage la relation entre art et langage dans l'œuvre de Xul Solar. Régine Cardis attire l'attention sur les œuvres abstraites, postérieures à 1958 et signées «J. C. Perinetti", que l'uruguayen José Cuneo réalisa à la fin de sa vie. Roberta Tenenini examine les enjeux esthétiques et politiques du mouvement non figuratif Madí (1944-1948). Enfin, ce dossier se termine avec des articles qui proposent un retour réflexif sur des moments dont la portée dépasse le seul champ artistique, indiquant des pistes de recherches innovantes sur le plan méthodologique ou sur les sujets traités. Au premier rang desquels Joaquín Torres García, pour sa trajectoire à rebours, ses expérimentations et son utopie. On le trouvera dans les sections «Reprises", où Maria Lucia Bastos Kern analyse comment le réseau symbolique du peintre est constitué de différentes tranches temporelles et mémorielles, sous-tendues par une quête d'ordre et de spiritualité. Sur le même artiste l'article d'Edgar Vidal, étudie de façon approfondie la singulière interrelation entre l'œuvre constructive et la pensée théosophique tout en explorant les possibilités de l'analyse lexicale appliquées aux livres d'art. Caroliny Pereira met en relation les héliographies de León Ferrari (série " Arquitectura de la locura ») avec l'exil au Brésil auquel il fut contraint pendant la dernière dictature argentine. Et Elena Lespes Munoz présente les recherches poétiques et plastiques de l'uruguayen Clemente Padín, un artiste peu étudié, et néanmoins important du Río de la Plata. 


\section{RÉSUMÉS}

Avec ce numéro consacré aux productions et propositions artistiques dans les pays du Río de la Plata au cours du XXe siècle - il ne concernera par ailleurs que les arts plastiques -, on a cherché à éviter les problématiques par trop « argentino- » ou « uruguayo-centrées ", celles-là même qui s'interrogent sur l'art «national » ou « international ». On a souhaité aussi éviter un panorama, même sélectif, des séquences classiques (écoles ou courants) et des chronologies canoniques (modernisme, avant-gardes, années des dictatures), au bénéfice d'études qui permettent de comprendre quelques enjeux esthétiques des pratiques artistiques, replacées dans leur contexte de production.

\section{AUTEURS}

\section{JACQUES POLONI-SIMARD}

EHESS-CERMA/Mondes américains

\section{EDGARD VIDAL}

CNRS-CRAL-EFISAL 